\title{
Análise Clínica e Epidemiológica de 348 Casos de Adenocarcinoma Prostático Atendidos em um Centro Oncológico de Referência no Maranhão, Brasil Clinical and Epidemiological Analysis of 348 Cases of Prostate Adenocarcinoma Attended in a Cancer Care Center in Maranhão, Brazil Análisis Clínico y Epidemiológico de 348 Casos de Adenocarcinoma de Próstata Tratados en un Centro de Referencia Oncológico en Maranhão, Brasil
}

\author{
Paulo Vitor Furtado Ribeiro ${ }^{1}$; Raimunda Ribeiro da Silva ${ }^{2}$; Kamilla Karolinne DallAgnol Torres dos Santos ${ }^{3}$; Flávia Helen Furtado Loureiro ${ }^{4}$; Priscilla \\ Furtado Amorim Franklin da Costa ${ }^{5}$ Lorena Martel Uruçu ${ }^{6}$; Anne Beatriz Sousa Carlos ${ }^{7}$ Thalita Marçal Machado ${ }^{8}$; Erica Fernanda Mesquita Galvez \\ Valdez ${ }^{9}$ Vinicius Batista Amaral10
}

\section{Resumo}

Introduçáo: $\mathrm{O}$ câncer de próstata é a principal neoplasia maligna no sexo masculino, depois do câncer de pele não melanoma. Informações clínicas e epidemiológicas sobre essa patologia no Maranhão são escassas. Objetivos: Analisar informaçóes clínicas e epidemiológicas de pacientes diagnosticados com câncer de próstata em um centro oncológico de referência no Maranhão, no período de janeiro de 2008 a dezembro de 2009. Método: Trata-se de um estudo retrospectivo e descritivo onde foram pesquisados 348 prontuários de pacientes com diagnóstico de câncer de próstata no Instituto Maranhense de Oncologia Aldenora Bello. As variáveis pesquisadas foram: faixa etária; procedência; ocupação; antígeno prostático específico inicial; escore de Gleason da biópsia; escore de Gleason da peça cirúrgica (em pacientes cujas terapêuticas incluíram prostatectomia radical ou ressecção transuretral de próstata); e tratamento instituído. Resultados: A faixa etária mais acometida foi de 71 a 80 anos (39,7\%). A maior parte dos pacientes era procedente de São Luís - MA (64,1\%) e não possuía ocupação (55,2\%). A maioria dos pacientes $(32,8 \%)$ apresentou antígeno prostático específico inicial entre 4,1 e $10 \mathrm{ng} / \mathrm{ml}$. O Gleason da biópsia revelou, em 48,5\% dos casos, tumores moderados a fracamente diferenciados. A terapêutica predominante foi composta de tratamento clínico e/ ou orquiectomia subcapsular bilateral. Conclusáo: $\mathrm{O}$ presente estudo demonstrou uma associaçáo significativa do antígeno prostático específico com a faixa etária e o Gleason, revelando maior tendência de aumento dos seus níveis quanto maior a idade e maior a graduação histológica dos tumores. Do total dos pacientes, $36,5 \%$ obtiveram escores de Gleason da biópsia e peça cirúrgica equivalentes.

Palavras-chave: Humanos; Masculino; Neoplasias da Próstata; Adenocarcinoma; Fatores Epidemiológicos; Biópsia

Trabalho realizado no Instituto Maranhense de Oncologia Aldenora Bello (IMOAB). São Luís (MA), Brasil.

${ }^{1}$ Médico graduado pela Universidade Ceuma. São Luís (MA), Brasil. E-mail: vfrpaulo@yahoo.com.br.

${ }^{2}$ Médica. Doutora em Fisiopatologia pela Universidade Estadual do Rio de Janeiro (UERJ). Especialista em Anatomia Patológica pelo Instituto de Assistência dos Servidores do Estado do Rio de Janeiro (IASERJ). São Luís (MA), Brasil. E-mail: raidaribeiro@hotmail.com.

${ }^{3}$ Médica graduada pela Universidade Ceuma. São Luís (MA), Brasil. E-mail: kamilladallagnol@yahoo.com.br.

${ }^{4}$ Odontóloga. Pós-Doutoranda em Saúde Coletiva na Universidade Federal do Maranhão (UFMA). Mestre e Doutora em Materiais Dentários pela

Faculdade de Odontologia de Piracicaba (FOP)/ UNICAMP. Graduada pela UFMA. São Luís (MA), Brasil. E-mail: flaviafurtado01@hotmail.com.

${ }^{5}$ Médica graduada pela UFMA. São Luís (MA), Brasil. E-mail: pri_furtado85@hotmail.com.

${ }^{6}$ Médica graduada pela Universidade Ceuma. São Luís (MA), Brasil. E-mail: lorena_martel@hotmail.com.

${ }^{7}$ Médica graduada pela Universidade Ceuma. São Luís (MA), Brasil. E-mail: annebeatriz19@gmail.com.

${ }^{8}$ Médica graduada pela Universidade Ceuma. São Luís (MA), Brasil. E-mail: thalitamm88@hotmail.com.

${ }^{9}$ Médica graduada pela Universidade Ceuma. São Luís (MA), Brasil. E-mail: ericamesquita11@hotmail.com.

${ }^{10}$ Médico graduado pela Universidade Ceuma. São Luís (MA), Brasil. E-mail: viniciusamaral1@hotmail.com.

Endereço para correspondência: Paulo Vitor Furtado Ribeiro. Rua Maracaçumé, Quadra 15, Casa 30 - Calhau. São Luís (MA), Brasil. CEP: 65071-590. 


\section{INTRODUÇÃO}

Para o Brasil, no ano de 2012, a estimativa foi de 60.180 casos novos de câncer de próstata $(\mathrm{CaP})$. Esses valores correspondem a um risco estimado de 62 casos novos a cada 100 mil homens. Nas regióes Sudeste (78/100 mil) e Nordeste (43/100 mil), o CaP é o mais incidente entre os homens. Sem considerar os tumores da pele não melanoma, é o mais frequente nas regiốes Centro-Oeste (75/100 mil), Sul $(68 / 100 \mathrm{mil})$ e Norte $(30 / 100 \mathrm{mil})^{1}$.

Os termos "câncer de próstata" e "adenocarcinoma prostático", quando usados sem classificaçôes, se referem à variante comum ou acinar do $\mathrm{CaP}^{2}$.

A idade é um marcador de risco importante uma vez que tanto a incidência quanto a mortalidade aumentam exponencialmente após os 50 anos. $\mathrm{O}$ risco de $\mathrm{CaP}$ aumenta uma vez e meia quando um parente de primeiro grau (pai ou irmão) tem o tumor, e cinco vezes quando são acometidos dois parentes de primeiro grau. Nos casos hereditários, o câncer manifesta-se mais precocemente, muitas vezes, antes dos 50 anos. A incidência e a taxa de mortalidade são maiores em negros do que em brancos norte-americanos e vêm aumentando com o decorrer do tempo. Um determinado tipo de dieta é apontado como outro fator de risco para desenvolver o câncer. Nesse sentido, a dieta rica em gordura saturada (especialmente gordura animal) e pobre em fibra aumenta seu risco. Como ocorre na hiperplasia nodular da próstata, acredita-se que os androgênios participem na patogênese do câncer. O suporte para essa tese geral reside na inibição desses tumores, que pode ser alcançada com a orquiectomia ${ }^{2,3,4,5}$.

De acordo com a Sociedade Brasileira de Urologia, recomenda-se a realização do rastreamento de $\mathrm{CaP}$ com exame do antígeno prostático específico (PSA) anual e toque retal em homens entre 50 e 80 anos. Nos homens negros ou com parentes de primeiro grau com diagnóstico de CaP, o rastreamento pode ser iniciado aos 45 anos $^{6}$.

De acordo com o Instituto Nacional de Câncer José Alencar Gomes da Silva (INCA), deve-se indicar o rastreamento oportunístico, ou seja, a sensibilizaçáo de homens com idade entre 50 e 70 anos, que procuram os serviços de saúde por motivos outros que o CaP, sobre a possibilidade de detecção precoce desse câncer por meio da realização de exames do toque retal e da dosagem do PSA total, informando-os sobre suas limitações, os benefícios e os riscos sobre a detecção precoce do $\mathrm{CaP}^{7}$.

O PSA é uma proteína produzida quase exclusivamente pelo epitélio prostático. Baixas concentraçóes do PSA são produzidas pelo endométrio, tecido mamário e carcinomas renais e adrenais, além de terem sido encontradas quantidades mensuráveis no soro feminino; no entanto, na prática clínica, o PSA é suficientemente específico para a glândula prostática. Embora o PSA seja órgão-específico, tal marcador não é câncer-específico. Algumas doenças como hiperplasia benigna da próstata também podem elevar o nível sérico de PSA ${ }^{8}$.

O PSA é considerado o marcador mais importante para rastrear, detectar, estagiar e monitorar o $\mathrm{CaP}^{9}$.

A biópsia da próstata guiada por ultrassonografia transretal é o método mais recomendado na prática para diagnosticar CaP. Vários autores têm tentado quantificar de diversas maneiras as informaçôes da biópsia prostática, na tentativa de identificar achados pré-operatórios que possam ajudar a diminuir o subestagiamento e prever extensōes extracapsulares ou falha biológica. Essas biópsias, quando adequadamente realizadas, são fontes de inúmeras informaçôes, pois podem ser usadas para o estudo da cápsula, tecidos periprostáticos, espaços perineurais, quantificação de tumor nos fragmentos, podendo estimar com isso a multifocalidade, o volume do tumor e a extensão extraprostática?.

Em geral, o $\mathrm{CaP}$ compromete as regióes posterior e lateral da zona periférica da próstata, sendo multifocal em $50 \%$ dos casos e estendendo-se frequentemente ao ápice. O volume tumoral usualmente está associado ao grau histológico, à extensão extraprostática, ao comprometimento de margens cirúrgicas e ao tempo de sobrevida. Por apresentar padróes arquiteturais variados e características citológicas próprias, o $\mathrm{CaP}$ tem sido graduado em diferentes sistemas, sendo o grau de Gleason um dos mais utilizados ${ }^{10}$.

De acordo com o sistema de Gleason, o CaP é estratificado em cinco graus com base nos padróes glandulares e grau de diferenciação visto em pequeno aumento. O grau 1 representa os tumores mais diferenciados, nos quais as glândulas neoplásicas têm aparência uniforme e redonda e são agrupadas em nódulos circunscritos. Em contraste, os tumores de grau 5 não apresentam diferenciação glandular e as células tumorais infiltram o estroma na forma de cordóes, folhetos ou ninhos. Os outros graus se situam entre estes. A maioria dos tumores contém mais de um padrão e, nesse caso, é designado um grau primário para o padrão dominante $\mathrm{e}$ um grau secundário para o padrão subdominante. Os dois graus numéricos são então somados para se obter um grau ou escore de Gleason combinado 2 .

Escores de Gleason são frequentemente combinados em grupos com comportamentos biológicos semelhantes: 2 a 4 representando o câncer bem diferenciado; 5 a 6 representando câncer de grau intermediário; 7 representando câncer de moderado a fracamente diferenciado; e 8 a 10 representando câncer de alto grau².

Para doença não metastática, são adotadas como alternativas de tratamento: prostatectomia radical, radioterapia e hormonioterapia. Para tratamento de doença metastática, recomendam-se, como manobra inicial: deprivação androgênica, orquiectomia ou análogo do hormônio liberador do hormônio luteinizante 
(LHRH). A quimioterapia antineoplásica é reservada para pacientes com neoplasia metastática refratária ao tratamento hormonal. A ressecção transuretral de próstata (RTU) pode ser indicada como tratamento paliativo, nos pacientes com sintomas obstrutivos. Nos pacientes com dor óssea localizada, pode ser indicada radioterapia externa ${ }^{6,11}$.

Este estudo tem como objetivo analisar informaçóes clínicas e epidemiológicas de pacientes diagnosticados com $\mathrm{CaP}$ em um Centro Oncológico de Referência no Maranhão, no período de janeiro de 2008 a dezembro de 2009, por meio da determinação da frequência da faixa etária, procedência e ocupação dos pacientes, correlação dos valores séricos iniciais de PSA (obtidos antes da realização da biópsia transretal guiada por ultrassom) com a faixa etária e com o escore de Gleason obtido na biópsia, correlação do escore de Gleason obtido na biópsia com o escore de Gleason obtido da análise da peça cirúrgica (nos pacientes submetidos à prostatectomia ou ressecção transuretral de próstata) e determinação da frequência dos tratamentos instituídos.

\section{MÉTODO}

Após registro e aprovação pelo Comitê de Ética em Pesquisa (CEP) da Universidade Ceuma, sob número de protocolo $00559 / 11$, foi realizado um estudo retrospectivo e descritivo que utilizou dados dos prontuários de pacientes com diagnóstico de $\mathrm{CaP}$ no Instituto Maranhense de Oncologia Aldenora Bello (IMOAB), no período de janeiro de 2008 a dezembro de 2009. Foram analisadas as seguintes variáveis: 1) faixa etária; 2) procedência; 3) ocupaçáo; 4) valor sérico inicial de PSA (obtido antes da realizaçáo de biópsia transretal guiada por ultrassom); 5) escore de Gleason obtido pela biópsia; 6) escore de Gleason obtido pela análise da peça cirúrgica (nos pacientes cujas terapêuticas incluíram prostatectomia radical (PR) e linfadenectomia ilíaco obturadora bilateral (LIOB) ou ressecção transuretral de próstata); e 7) tratamento instituído.

A variável faixa etária foi estratificada em seis grupos: 1) $\leq 40$ anos; 2) 41 a 50 anos; 3) 51 a 60 anos; 4) 61 a 70 anos; 5) 71 a 80 anos e 6$) \geq 81$ anos. A variável procedência foi estratificada em três grupos: 1) São Luís - MA; 2) Interior do MA e 3) outros Estados. A variável ocupação foi estratificada em cinco grupos: 1) sem ocupação; 2) funcionários públicos; 3) profissionais liberais; 4) motoristas e 5) outras ocupaçóes. A variável PSA foi estratificada em cinco grupos: 1) $0 \mathrm{a} 4 \mathrm{ng} / \mathrm{ml}$; 2) 4,1 a $10 \mathrm{ng} / \mathrm{ml}$; 3) 10,1 a $20 \mathrm{ng} / \mathrm{ml}$; 4) 20,1 a $50 \mathrm{ng} / \mathrm{ml}$; e 5) > $50 \mathrm{ng} / \mathrm{ml}$. A variável escore de Gleason obtida da biópsia foi estratificada em quatro grupos: 1) 2 a 4 (tumor bem diferenciado); 2) 5 a 6 (tumor intermediário); 3) 7 (tumor de moderado a fracamente diferenciado) e 4) 8 a 10 (tumor de alto grau). A variável escore de Gleason obtida da análise da peça cirúrgica foi estratificada em cinco grupos: 1) 2 a 4 (tumor bem diferenciado); 2) 5 a 6 (tumor intermediário); 3) 7 (tumor de moderado a fracamente diferenciado); 4) 8 a 10 (tumor de alto Grau); e 5) não foi realizado (grupo dos pacientes cujas terapêuticas não incluíram $\mathrm{PR}$ e LIOB ou ressecção transuretral de próstata). A variável tratamento instituído foi estratificada em nove grupos: 1) prostatectomia radical (PR) + LIOB; 2) $\mathrm{PR}+\mathrm{LIOB}$ + tratamento clínico (radioterapia e/ou hormonioterapia e/ou quimioterapia); 3) $\mathrm{PR}+\mathrm{LIOB}+$ orquiectomia subcapsular bilateral (OSB); 4) PR + LIOB + tratamento clínico + OSB; 5) RTU; 6) RTU + tratamento clínico; 7) RTU + OSB; 8) RTU + tratamento clínico + OSB e 9) tratamento clínico e/ou OSB.

Os dados foram avaliados pelo programa SPSS for Windows 17.0 (2007). Inicialmente, foram realizadas as análises da estatística descritiva, ou seja, através de tabelas de frequência das variáveis estudadas. Posteriormente, foi feita a correlação não paramétrica de Spearman das variáveis: faixa etária, PSA, escore de Gleason da biópsia e escore de Gleason da peça cirúrgica. Depois, para se avaliar a associação das variáveis classificatórias (faixa etária e escore de Gleason da biópsia) com o nível de PSA e a associação da variável escore de Gleason da biópsia com o escore de Gleason da peça cirúrgica, foi realizado o teste náo paramétrico de qui-quadrado de independência $\left(\chi^{2}\right)$. O nível de significância para se rejeitar a hipótese de nulidade foi de $5 \%$, ou seja, considerou-se como estatisticamente significante um valor de $p<0,05$. $\mathrm{O}$ estudo dispensou o termo de consentimento livre e esclarecido (TCLE) por tratar-se de uma pesquisa feita com o uso de dados de prontuários do IMOAB, os quais foram mantidos em sigilo, conforme prevê os termos da Resolução 196/96 do Conselho Nacional de Saúde.

\section{RESULTADOS}

Foram pesquisados 401 prontuários no IMOAB que representavam todos os casos de $\mathrm{CaP}$ diagnosticados naquela Instituição, no período de janeiro de 2008 a dezembro de 2009. Devido à insuficiência de dados no prontuário, abandono de tratamento ou óbito, 53 pacientes foram excluídos do estudo. Avaliaram-se então 348 pacientes diagnosticados com CaP naquele período. A Tabela 1 demonstra a análise da frequência das variáveis (faixa etária, procedência, ocupação, PSA, escore de Gleason da biópsia, tratamento instituído e escore de Gleason da peça cirúrgica). Com relação à faixa etária dos pacientes, que variou de 40 a 90 anos (média de 68,6 anos, com desvio-padrão $\pm 8,48$ ), foi demonstrado que a maior parte dos pacientes encontrava-se na faixa etária de 71 a 80 anos (39,7\%). Quanto à procedência, a maioria $(\mathrm{n}=223 ; 64,1 \%)$ era de São Luís - MA. Com relação à ocupaçáo, a maior parte $(\mathrm{n}=192 ; 55,2 \%)$ era composta de indivíduos sem ocupação. 
Com relaçáo ao PSA, que variou de $0,1 \mathrm{ng} / \mathrm{ml}$ a $>1.000$ $\mathrm{ng} / \mathrm{ml}$ (média de 43,9 ng/ml, com desvio-padrão $\pm 67,86$ ), observou-se que a maioria dos pacientes $(32,8 \%)$ tinha PSA entre 4,1 e $10 \mathrm{ng} / \mathrm{ml}$. Através do escore de Gleason da biópsia, foi evidenciado na maior parte dos pacientes tumores de moderados a fracamente diferenciados (48,5\%). Com relação ao escore de Gleason obtido da peça cirúrgica, este não foi realizado na maior parte dos pacientes $(n=168 ; 48,3 \%)$ porque suas terapêuticas não incluíram PR + LIOB ou RTU.

De acordo com a Tabela 2, através da correlação não paramétrica de Spearman das variáveis: faixa etária, PSA, escore de Gleason da biópsia e escore de Gleason da peça cirúrgica, observou-se uma correlação baixa, positiva e significativa entre PSA e a faixa etária $(p<0,05)$, PSA e Gleason da biópsia $(p<0,01)$ e PSA e Gleason da peça cirúrgica $(p<0,01)$. Além disso, foi observada uma correlação média, positiva e significativa entre o Gleason da biópsia e o Gleason da peça cirúrgica $(p<0,01)$.

$\mathrm{Na}$ análise estatística com aplicação do teste náo paramétrico de qui-quadrado de independência das variáveis em relação ao PSA, considerando o nível de significância para se rejeitar a hipótese de nulidade de $5 \%$, ou seja, considerando-se como estatisticamente significante um valor de $p<0,05$, foi encontrada uma associação significativa com a variável faixa etária

Tabela 1. Distribuição de frequência das variáveis analisadas

\begin{tabular}{|c|c|c|c|c|c|}
\hline Variável & $\mathbf{N}$ & $\%$ & Variável & $\mathbf{N}$ & $\%$ \\
\hline \multicolumn{3}{|l|}{ Faixa etária } & \multicolumn{3}{|l|}{ Gleason da biópsia } \\
\hline$\leq 40$ & 1 & 0,3 & Tumor bem diferenciado (escore entre 2-4) & 6 & 1,7 \\
\hline $41-50$ & 5 & 1,4 & Tumor intermediário (escore entre 5-6) & 98 & 28,2 \\
\hline $51-60$ & 56 & 16,1 & $\begin{array}{l}\text { Tumor de moderado a fracamente } \\
\text { diferenciado (escore 7) }\end{array}$ & 169 & 48,5 \\
\hline $61-70$ & 129 & 37,1 & Tumor de alto grau (escore entre 8-10) & 75 & 21,6 \\
\hline $71-80$ & 138 & 39,7 & & & \\
\hline \multirow[t]{2}{*}{$\geq 81$} & 19 & 5,4 & Tratamento instituído & & \\
\hline & & & $\mathrm{PR}+\mathrm{LIOB}$ & 75 & 21,6 \\
\hline Procedência & & & PR + LIOB + TRATAMENTO CLÍNICO & 74 & 21,3 \\
\hline São Luís (MA) & 223 & 64,1 & $P R+L I O B+O S B$ & 1 & 0,3 \\
\hline Interior do MA & 123 & 35,3 & PR + LIOB + TRATAMENTO CLÍNICO + OSB & 1 & 0,3 \\
\hline \multirow[t]{2}{*}{ Outros Estados } & 2 & 0,6 & RTU & 5 & 1,4 \\
\hline & & & RTU + TRATAMENTO CLÍNICO & 13 & 3,7 \\
\hline Ocupação & & & RTU + OSB & 9 & 2,6 \\
\hline Sem ocupação & 192 & 55,2 & RTU + TRATAMENTO CLÍNICO + OSB & 4 & 1,2 \\
\hline Funcionários públicos & 15 & 4,3 & TRATAMENTO CLÍNICO E/OU OSB & 166 & 47,6 \\
\hline Profissionais liberais & 13 & 3,7 & & & \\
\hline Motoristas & 4 & 1,2 & Gleason da peça cirúrgica & & \\
\hline \multirow[t]{2}{*}{ Outras ocupações } & 124 & 35,6 & Tumor bem diferenciado (escore entre 2-4) & 1 & 0,3 \\
\hline & & & Tumor intermediário (escore entre 5-6) & 58 & 16,7 \\
\hline PSA (ng/ml) & & & $\begin{array}{l}\text { Tumor de moderado a fracamente } \\
\text { diferenciado (escore 7) }\end{array}$ & 81 & 23,2 \\
\hline $0-4$ & 20 & 5,7 & Tumor de alto grau (escore entre 8-10) & 40 & 11,5 \\
\hline $4,1-10$ & 114 & 32,8 & Não foi realizado & 168 & 48,3 \\
\hline $10,1-20$ & 85 & 24,4 & & & \\
\hline $20,1-0$ & 76 & 21,8 & Total & 348 & 100,0 \\
\hline$>50$ & 53 & 15,3 & & & \\
\hline Total & 348 & 100,0 & & & \\
\hline
\end{tabular}

Na variável procedência, o item referente ao interior do MA incluiu os seguintes municípios, cidades ou povoados (em ordem alfabética): Açailândia, Anajatuba, Arame, Axixá, Bacabal, Barreirinhas, Bequimão, Buriti Bravo, Cajapió, Caxias, Chapadinha, Colinas, Conceição do Lago, Coroatá, Cururupu, Esperantinópolis, Formosa da Serra Negra, Grajaú, Guimarães, Humberto de Campos, Icatu, Itapecuru Mirim, Lago da Pedra, Lagoa Grande do Maranhāo, Mata Roma, Matinha, Morros, Paço do Lumiar, Panaquatira, Paraibano, Pastos Bons, Pedreiras, Pedro do Rosário, Penalva, Pinheiro, Pio XII, Poção de Pedras, Porto Rico do Maranhão, Presidente Juscelino, Riachão, Rosário, Samambaia, Santa Helena, Santa Inês, Santa Luzia, Santa Quitéria do Maranhão, Santa Rita, São Benedito do Rio Preto, São Bento, São Domingos do Azeitão, São José de Ribamar, São Mateus do Maranhão, São Vicente Ferrer, Serra Negra, Sucupira do Norte, Trizidela do Vale, Turilândia, Urbano Santos, Vargem Grande, Viana e Vitória do Mearim. O item referente aos outros estados incluiu (em ordem alfabética): Belém (PA) e Macapá (AP). Item referente às outras ocupaçóes incluiu (em ordem alfabética): auxiliares de higiene, carroceiros, feirantes, lavradores, militares, pescadores e vigilantes. Legendas: PR (prostatectomia radical), LIOB (linfadenectomia ilíaco obturadora bilateral), tratamento clínico (radioterapia e/ou hormonioterapia e/ou quimioterapia), OSB (orquiectomia subcapsular bilateral) e RTU (ressecção transuretral de próstata) 
$(p=0,009)$ e escore de Gleason da biópsia $(p<0,0001)$, conforme demonstrado na Tabela 3 . Foi encontrada também uma associação significativa $(p<0,0001)$ entre as variáveis: escore de Gleason da biópsia e escore de Gleason da peça cirúrgica, conforme demonstrado na Tabela 4.

Tabela 2. Correlação de Spearman

\begin{tabular}{l|c|c|c|c}
\hline & Faixa etária & PSA & $\begin{array}{c}\text { Gleason da } \\
\text { biópsia }\end{array}$ & $\begin{array}{c}\text { Gleason da peça } \\
\text { cirúrgica }\end{array}$ \\
\hline Faixa etária & 1.000 & $0,133^{*}$ & $-0,002$ & 0,063 \\
\hline PSA & $0,133^{*}$ & 1.000 & $0,292^{* *}$ & $0,231^{* *}$ \\
\hline Gleason da biópsia & $-0,002$ & $0,292^{* *}$ & 1.000 & $0,638^{* *}$ \\
\hline Gleason da peça cirúrgica & 0,063 & $0,231^{* *}$ & $0,638^{* *}$ & 1.000 \\
\hline
\end{tabular}

${ }^{*} \mathrm{p}<0,05 \quad{ }^{* *} \mathrm{p}<0,01$

Tabela 3. Teste do qui-quadrado de independência das variáveis: faixa etária e escore de Gleason da biópsia em relação ao nível de PSA

\begin{tabular}{|c|c|c|c|c|c|c|c|}
\hline \multicolumn{8}{|c|}{ PSA (ng/ml) } \\
\hline Variável & $0-4$ & $4,1-10$ & $10,1-20$ & $20,1-50$ & $>50$ & Total & $\boldsymbol{p}$ \\
\hline \multicolumn{8}{|l|}{ Faixa etária } \\
\hline$\leq 40$ & $0(0,0)$ & $0(0,0)$ & $0(0,0)$ & $0(0,0)$ & $1(1,9)$ & $1(0,3)$ & 0,009 \\
\hline $41-50$ & $0(0,0)$ & $1(0,9)$ & $0(0,0)$ & $3(3,9)$ & $1(1,9)$ & $5(1,4)$ & \\
\hline $51-60$ & $4(20,0)$ & $29(25,4)$ & $11(12,9)$ & $6(7,9)$ & $6(11,3)$ & $56(16,1)$ & \\
\hline $61-70$ & $7(35,0)$ & $37(32,5)$ & $43(50,6)$ & $28(36,8)$ & $14(26,5)$ & $129(37,1)$ & \\
\hline $71-80$ & $7(35,0)$ & $44(38,6)$ & $26(30,6)$ & $36(47,5)$ & $25(47,1)$ & $138(39,7)$ & \\
\hline$\geq 81$ & $2(10,0)$ & $3(2,6)$ & $5(5,9)$ & $3(3,9)$ & $6(11,3)$ & $19(5,4)$ & \\
\hline \multicolumn{8}{|l|}{ Gleason da biópsia } \\
\hline Tumor bem diferenciado & $0(0,0)$ & $3(2,6)$ & $1(1,2)$ & $2(2,6)$ & $0(0,0)$ & $6(1,7)$ & $<0,0001$ \\
\hline Tumor intermediário & $9(45,0)$ & $42(36,8)$ & $30(35,3)$ & $9(11,9)$ & $8(15,1)$ & $98(28,2)$ & \\
\hline $\begin{array}{l}\text { Tumor de moderado a } \\
\text { fracamente diferenciado }\end{array}$ & $7(35,0)$ & $57(50,0)$ & $41(48,2)$ & $41(53,9)$ & $23(43,4)$ & $169(48,5)$ & \\
\hline Tumor de alto grau & $4(20,0)$ & $12(10,6)$ & $13(15,3)$ & $24(31,6)$ & $22(41,5)$ & $75(21,6)$ & \\
\hline Total & $20(100,0)$ & $114(100,0)$ & $85(100,0)$ & $76(100,0)$ & $53(100,0)$ & $348(100,0)$ & \\
\hline
\end{tabular}

Valores de escore de Gleason entre 2-4 (para tumores bem diferenciados), 5-6 (para tumores intermediários), 7 (para tumores de moderados a fracamente diferenciados) e 8-10 (para tumores de alto grau)

Tabela 4. Teste do qui-quadrado de independência das variáveis: escore de Gleason da biópsia e escore de Gleason da peça cirúrgica

\begin{tabular}{l|c|c|c|c|c|c|}
\hline \multicolumn{7}{|c|}{ Gleason da biópsia } \\
\hline \multicolumn{1}{|c|}{ Gleason da peça } & $\begin{array}{c}\text { Tumor bem } \\
\text { diferenciado }\end{array}$ & $\begin{array}{c}\text { Tumor } \\
\text { intermediário } \\
\text { moderado a } \\
\text { fracamente } \\
\text { diferenciado }\end{array}$ & $\begin{array}{c}\text { Tumor de } \\
\text { alto grau }\end{array}$ & Total \\
\hline Tumor bem diferenciado & $1(16,7)$ & $0(0,0)$ & $0(0,0)$ & $0(0,0)$ & $1(0,3)$ & $<0,0001$ \\
\hline Tumor intermediário & $2(33,3)$ & $33(33,7)$ & $20(11,8)$ & $3(4,0)$ & $58(16,7)$ \\
\hline $\begin{array}{l}\text { Tumor de moderado a } \\
\text { fracamente diferenciado }\end{array}$ & $0(0,0)$ & $18(18,4)$ & $61(36,1)$ & $2(2,7)$ & $81(23,2)$ \\
\hline Tumor de alto grau & $0(0,0)$ & $2(2,0)$ & $6(3,6)$ & $32(42,7)$ & $40(11,5)$ \\
\hline Não foi realizado & $3(50,0)$ & $45(45,9)$ & $82(48,5)$ & $38(50,6)$ & $168(48,3)$ \\
\hline Total & $6(100,0)$ & $98(100,0)$ & $169(100,0)$ & $75(100,0)$ & $348(100,0)$ \\
\hline
\end{tabular}

Valores de escore de Gleason entre 2-4 (para tumores bem diferenciados), 5-6 (para tumores intermediários), 7 (para tumores de moderados a fracamente diferenciados) e 8-10 (para tumores de alto grau) 
Analisando-se a relação entre PSA e faixa etária, de acordo com a Tabela 3, considerando o subgrupo dos pacientes com PSA entre 0 e $4 \mathrm{ng} / \mathrm{ml}(\mathrm{n}=20 ; 100 \%)$, observou-se que $\mathrm{n}=7$ (35\%) encontravam-se entre 61 e 70 anos e $n=7$ (35\%) entre 71 e 80 anos. Considerando o subgrupo dos pacientes com PSA entre 4,1 e $10 \mathrm{ng} / \mathrm{ml}$ $(\mathrm{n}=114 ; 100 \%)$, evidenciou-se que a maior parte $(\mathrm{n}=44$; $38,6 \%)$ encontrava-se entre 71 e 80 anos. Considerando o subgrupo dos pacientes com PSA entre 10,1 e $20 \mathrm{ng} /$ $\mathrm{ml}(\mathrm{n}=85 ; 100 \%)$, observou-se que a maioria $(\mathrm{n}=43$; $50,6 \%$ ) encontrava-se entre 61 e 70 anos. Considerando o subgrupo de pacientes com PSA entre 20,1 e $50 \mathrm{ng} / \mathrm{ml}$ $(\mathrm{n}=76 ; 100 \%)$, evidenciou-se que a maior parte $(\mathrm{n}=36$; $47,5 \%)$ encontrava-se entre 71 e 80 anos. Considerando o subgrupo de pacientes com PSA igual ou maior que 50 $\mathrm{ng} / \mathrm{ml}(\mathrm{n}=53 ; 100 \%)$, observou-se que a maioria $(\mathrm{n}=25$; $47,1 \%$ ) encontrava-se entre 71 e 80 anos.

Analisando-se a relação entre PSA e escore de Gleason da biópsia, de acordo com a Tabela 3, foi observado para todos os subgrupos de PSA (entre $0-4 \mathrm{ng} / \mathrm{ml}$; entre 4,1-10 $\mathrm{ng} / \mathrm{ml}$; entre $10,1-20 \mathrm{ng} / \mathrm{ml}$; entre $20,1-50 \mathrm{ng} / \mathrm{ml}$ e > $50 \mathrm{ng} / \mathrm{ml}$ ) que a maior parte dos pacientes apresentou tumores de moderados a fracamente diferenciados.

Analisando-se a Tabela 4, considerando o total de pacientes analisados $(n=348 ; 100 \%)$, observou-se que $\mathrm{n}=180(51,7 \%)$ obtiveram tanto o escore do Gleason da biópsia como o da análise de suas peças cirúrgicas, pois $\mathrm{PR}$ + LIOB ou RTU fez parte da terapêutica desse subgrupo. Evidenciou-se que $\mathrm{n}=127(36,5 \%)$ obtiveram valores do escore de Gleason da biópsia e da peça equivalentes (grau de diferenciação histopatológica semelhante), enquanto $\mathrm{n}=53(15,3 \%)$ obtiveram valores do escore de Gleason da biópsia e da peça divergentes (grau de diferenciação histopatológica sub ou superestimados pela biópsia).

Com relação ao tratamento instituído, de acordo com a Tabela 1, observou-se que a maior parte dos pacientes $(\mathrm{n}=166 ; 47,6 \%)$ foi submetida ao tratamento clínico e/ ou OSB.

\section{DISCUSSÃO}

Em pesquisas realizadas por Cambruzzi et al.; Migowski e Silva, nos Estados do Rio Grande do Sul e Rio de Janeiro, respectivamente, um percentual elevado de CaP foi encontrado na faixa etária de 60-69 anos, representando $44,9 \%$ e $48,4 \%$ de suas amostras ${ }^{10,12}$. No presente estudo, foi demonstrado que a maior parte dos pacientes acometidos (39,7\%) tinha entre 71 e 80 anos de idade, revelando, no total de pacientes analisados, uma frequência maior do $\mathrm{CaP}$ em uma faixa etária mais avançada. Tal achado é semelhante ao encontrado por Penaforte et al., que evidenciaram nos Estados de Pernambuco e Bahia uma maior concentração de pacientes com CaP na faixa de 70 a 79 anos, representando $36,3 \%$ de sua população analisada ${ }^{13}$. Os seguintes achados, embora não confirmem, sugerem um diagnóstico tardio do $\mathrm{CaP}$ no Nordeste quando comparado ao Sudeste e Sul do país.

Evidenciou-se que grande parte dos pacientes $(64,1 \%)$ era procedente da capital do Estado (São Luís). A explicaçáo para tal fato pode se encontrar na maior facilidade de acesso ao Centro Oncológico pelos pacientes provenientes da capital do que os residentes em locais mais distantes, como em cidades do interior ou outros estados.

Mais da metade dos pacientes $(55,2 \%)$ foi representada pelo subgrupo dos indivíduos sem ocupação. Segundo o Ministério da Previdência Social, têm direito ao benefício da aposentadoria por idade os trabalhadores urbanos do sexo masculino a partir dos 65 anos e do sexo feminino a partir dos 60 anos de idade. Os trabalhadores rurais podem pedir aposentadoria por idade com cinco anos a menos: a partir dos 60 anos, homens; e a partir dos 55 anos, mulheres ${ }^{14}$. Partindo desse pressuposto, observa-se que o estudo apresenta coerência em tal quesito, já que a maioria dos homens pesquisados, por meio de prontuários, apresentou idade superior a 65 anos (entre 71 e 80 anos).

Em estudo realizado no Rio Grande do Sul, Dini e Koff analisaram a distribuição do CaP por faixa de PSA e observaram que $93,8 \%$ dos casos de sua amostra apresentaram PSA $>4 \mathrm{ng} / \mathrm{ml}$, sendo $53,8 \%$ na faixa entre 4-10 $\mathrm{ng} / \mathrm{ml}^{15}$. O presente estudo demonstrou resultado semelhante, evidenciando que a maior parte dos pacientes $(32,8 \%)$ apresentava PSA entre 4,1 e $10 \mathrm{ng} / \mathrm{ml}$.

Escores de Gleason de 2 a 4 sáo tipicamente encontrados em tumores pequenos na zona de transição. Em amostras cirúrgicas, esse câncer de baixo grau é tipicamente um achado incidental na ressecção transuretral realizada para sintomas de hiperplasia prostática benigna. A maioria dos cânceres potencialmente tratáveis, que são detectados à biópsia por agulha, apresenta escores de Gleason de 5 a 7. Tumores com escores de Gleason de 8 a 10 tendem a ser cânceres avançados com pouca probabilidade de cura² . No presente estudo, foi evidenciado, através da biópsia prostática, que a maior parte dos pacientes $(48,5 \%)$ apresentou escore de Gleason $=7$ (tumores de moderados a fracamente diferenciados), inferindo em tais casos a possibilidade de boa resposta ao tratamento.

Analisando a distribuição da faixa etária de acordo com os níveis de PSA, foi observado que os níveis de PSA dependem da faixa etária dos pacientes, ou seja, que os níveis de PSA nos pacientes com CaP tendem a aumentar com a idade $(p=0,009)$. Tal achado é corroborado por Dini, que afirma a existência de correlação direta entre o valor do PSA com neoplasia ${ }^{16}$.

Analisando a distribuição do escore de Gleason obtido da biópsia prostática com os níveis de PSA, observou-se que os níveis do escore de Gleason possuem associação altamente significativa com os níveis de PSA, ou seja, na 
presente amostra quanto maiores os níveis de PSA maior a tendência de tais tumores a serem indiferenciados $(\mathrm{p}<0,0001)$. A pesquisa de Toniazzo corrobora tal achado e sugere que pacientes com níveis séricos de PSA aumentados apresentem tumores de maior volume e menos diferenciados histologicamente ${ }^{17}$.

Dos sujeitos analisados, 48,3\% dos pacientes não realizaram o escore de Gleason da peça cirúrgica, pois a PR + LIOB ou RTU não foram incluídos em seu tratamento. Analisando-se o subgrupo dos 180 pacientes que obtiveram ambos os escores de Gleason (da biópsia e da peça), em 70,6\% dos casos houve concordância e 29,4\% discrepância, sendo subgraduados em 15,5\% e supergraduados em 13,9\%. Cury em seu estudo analisou 120 pacientes com CaP nos estádios clínicos T1, T2 e T3a submetidos à linfadenectomia ilíaca e à cirurgia radical da próstata. Em tal estudo, foram analisadas a concordância e a discrepância na comparação entre a biópsia de próstata e o espécimen cirúrgico, havendo concordância em 32,5\% dos pacientes, subgraduação em $62,5 \%$ e supergraduação em $5 \%{ }^{18}$. Antonopoulos, por sua vez, analisando 46 pacientes e estratificando os tumores diagnosticados em bem diferenciados (Gleason 2-4); moderadamente diferenciados (Gleason 5 e 6); e indiferenciados (Gleason 7-10), observou que, em 60,9\% dos casos, houve coincidência de classificação entre biópsia e peça cirúrgica, em 39,9\% dos casos subestimação e em $0,2 \%$ dos casos superestimação ${ }^{19}$.

Com relação ao tratamento, as informações encontradas coincidem com as da literatura, havendo poucas diferenças não significativas estatisticamente.

\section{CONCLUSÃO}

Este estudo demonstrou maior ocorrência de $\mathrm{CaP}$ em pacientes com idade entre 71 e 80 anos, sem ocupação e procedentes de São Luís.

A correlação entre faixa etária e os valores de PSA obtidos antes da realização de biópsia transretal permitiu concluir, na presente amostra, que tais valores tendem a aumentar com a idade e também com o grau de indiferenciação dos tumores.

Dos pacientes submetidos à cirurgia, a maior parte obteve escores de Gleason da biópsia e peça cirúrgica equivalentes.

A maioria dos pacientes foi submetida ao tratamento clínico e/ou orquiectomia subcapsular bilateral.

\section{AGRADECIMENTOS}

Ao Doutor Sílvio José Lima Moreira, Médico Urologista do Centro de Urologia do Maranhão e Professor da Universidade Ceuma e ao Doutor George Castro Figueira de Mello, Médico Patologista do Instituto
Maranhense de Oncologia Aldenora Bello e Professor da Universidade Ceuma, pelo incentivo ao estudo sobre o tema do artigo. À Professora Cássia Cordeiro Furtado, Doutora em Informação e Comunicação em Plataformas Digitais, pelas sugestôes propostas na elaboração do texto final do artigo. Aos Senhores Andrey Pinto da Silva e Antônio Euzébio Campelo Neto, funcionários do Setor de Arquivos do Instituto Maranhense de Oncologia Aldenora Bello, pela disponibilização dos dados em prontuário necessários para realização deste estudo.

\section{CONTRIBUIÇÕES}

Paulo Vitor Furtado Ribeiro contribuiu na concepção e no planejamento de estudo; na obtenção, análise e interpretação dos dados; assim como na elaboração do texto final do artigo. Raimunda Ribeiro da Silva contribuiu na concepçáo e planejamento do projeto de pesquisa, assim como na redação e revisão crítica do texto final do artigo. Kamilla Karolinne Dall Agnol Torres dos Santos contribuiu na concepção e planejamento do projeto de pesquisa e na obtenção e análise dos dados em prontuário. Flávia Helen Furtado Loureiro, Priscilla Furtado Amorim Franklin da Costa, Thalita Marçal Machado, Lorena Martel Uruçu, Anne Beatriz Sousa Carlos, Erica Fernanda Mesquita Galdez Valdez e Vinicius Batista Amaral contribuíram na obtenção dos dados em prontuário.

\section{Declaração de Conflito de Interesses: Nada a Declarar. \\ REFERÊNCIAS}

1. Instituto Nacional de Câncer José Alencar Gomes da Silva. Estimativa 2012: incidencia de câncer no Brasil [Internet]. Rio de Janeiro: INCA; 2012 [acesso 2012 Mai 17]. Disponível em: http:// http://www1.inca.gov. br/estimativa/2012/.

2. Kumar V, Abbas K, Fausto N. Robbins e Cotran: patologia - bases patológicas das doenças. Rio de Janeiro: Elsevier; 2005.

3. Gomes R, Rebello LEFS, Araújo FC, Nascimento EF. A prevenção do câncer de próstata: uma revisão da literatura. Ciênc Saúde Coletiva. 2008; 13(1): 235-46.

4. Gonçalves TN, Mella Junior SE. Avaliação do nível sérico de antígeno prostático específico (PSA) em pacientes da cidade de Campo Mourão - PR. Rev Bras Anal Clin. 2007; 39(4):279-81.

5. Schroder FH. Screening for prostate cancer. Urology Clinic North Am 2003; 30(27): 239-51.

6. Sociedade Brasileira de Urologia. Diretrizes urooncologia: câncer de próstata [Internet]. 2012 [acesso 2012 Mai 19]. Disponível em: http://www.sbu.org. $\mathrm{br} /$ ?diretrizes. 
7. Instituto Nacional do Câncer (Brasil). Programa nacional de controle do câncer da próstata: documento de consenso. Rio de Janeiro: INCA; 2002.

8. Van Der Cruijsen-Koeter IW, Wildhagen MF, De Koning HJ, Schroder FH. The value of current diagnostic tests in prostate cancer screening. BJU International 2001; 88(5): 458-66.

9. Calvete AC, Srougi M, Nesrallah LJ, Dall'Oglio MF, Ortiz V. Avaliação da extensão da neoplasia em câncer de próstata: valor do PSA, da percentagem de fragmentos positivos e da escala de Gleason. Rev Assoc Méd Bras. 2003; 49(3): 250-4.

10. Cambruzzi E; Zettler CG; Pegas KL; Teixeira SL. Relação entre escore de Gleason e fatores prognósticos no adenocarcinoma acinar de próstata. J Bras Patol Med Lab. 2010; 46(1): 61-8.

11. Passeroti CC, Carvalhal GF, Almeida JC, Fonseca L, Nogueira L, Tobias-Machado M, et al. Diretrizes do tratamento cirúrgico do câncer de próstata [Internet]. 2012 [acesso 2012 Jun 23]. Disponível em: http://www. saudedireta.com.br/docsupload/1335443528diretrizesd otratamentodocancerdeprostata.pdf.

12. Migowski A, Silva GA. Sobrevida e fatores prognósticos de pacientes com câncer de próstata clinicamente localizado. Rev Saúde Pública. 2010; 44(2): 344-52.

13. Penaforte ACC, Moura HSS, Gois LPBL, Alcantara DL, Almeida Neto A. Estudo epidemiológico dos casos diagnosticados de câncer de próstata na região do submédio São Francisco no período de 2005 a 2009 [dissertação]. Pernambuco: Universidade de Pernambuco; 2009.

14. Ministério da Previdência Social (Brasil). Aposentadoria por idade [Internet]. 2012 [acesso 2012 Jul 26]. Disponível em: http://www.previdencia.gov.br/conteudoDinamico. php?id $=15$.

15. Dini LI, KoffWJ. Perfil do câncer de próstata no hospital de clínicas de Porto Alegre. Rev Ass Med Bras. 2005; 52(1): 28-31.

16. Dini LI. Perfil do câncer de próstata em um programa de rastreamento na cidade de Porto Alegre [tese]. Rio Grande do Sul: Universidade Federal do Rio Grande do Sul; 2002.

17. Toniazzo GP. Correlação entre níveis séricos de PSA e estimativa do volume tumoral em fragmentos de biópsia de próstata em pacientes portadores de adenocarcinoma da próstata [tese]. Rio Grande do Sul: Universidade Federal do Rio Grande do Sul; 2005.

18. Cury J, Srougi M, Leite KRM, Lopes LHC, Carneiro PC. Correlação entre a graduação histológica de biópsias e do espécimen cirúrgico em câncer de próstata. Rev Col Bras Cir. 1999; 26(1): 21-5.

19. Antonopoulos IM, Pompeo ACL, Saldanha LB, Arap MA, Danilovic A, Arap S. Gleason score. Comparative study between transrectal prostate biopsy and radical prostatectomy specimen. Braz J Urol. 2000; 26(6): 609-13. 


\section{Abstract}

Introduction: The prostate cancer is the main malignant neoplasm that occurs in men after nonmelanoma skin cancer. Clinical and epidemiological information on this pathology are scarce in Maranhão. Objectives: Analysis of clinical and epidemiological information of patients diagnosed with prostate cancer, in a Cancer Care Reference Center in Maranhão, between january 2008 and december 2009. Method: This is a retrospective and descriptive study in which 348 medical records of patients diagnosed with prostate cancer, at the Instituto Maranhense de Oncologia Aldenora Bello, were surveyed. The variables under consideration were: age, origin, occupation, initial prostate-specific antigen, Gleason score of the biopsy, Gleason score of the surgical specimen (in patients whose treatment included radical prostatectomy or transurethral resection of the prostate), and treatment. Results: The most affected age group was from 71 to 80 years old (39.7\%). Most of patients were from São Luís - MA (64.1\%) and did not have occupation (55.2\%). Most of patients (32.8\%) had prostate-specific antigen between 4.1 and $10 \mathrm{ng} / \mathrm{ml}$. The Biopsy Gleason revealed moderate to weakly differentiated tumors in $48.5 \%$ of the cases. The predominant therapy was clinical treatment and/or subcapsular bilateral orquiectomy. Conclusion: This study demonstrated a significant association between the prostate-specific antigen and the age and Gleason Score, revealing in these patients a greater tendency to increase their levels the greater the age and higher histologic grading of tumors. A percentage of $36.5 \%$ of patients had both Biopsy and Surgery Specimen Gleason Scores equivalents.

Key words: Humans; Male; Prostatic Neoplasms; Adenocarcinoma; Epidemiologic Factors; Biopsy

\section{Resumen}

Introducción: El cáncer de próstata es la principal neoplasia maligna en los varones después del cáncer de piel no melanoma. Informaciones clínicas y epidemiológicas sobre esta enfermedad en Maranhão son escasas. Objetivos: Analizar información clínica y epidemiológica de pacientes con diagnóstico de cáncer de próstata tratados en un Centro de Referencia Oncológico en Maranhão, de enero de 2008 a diciembre de 2009. Método: Se trata de un estudio retrospectivo y descriptivo donde se analizaron 348 historiales clínicos de pacientes diagnosticados con cáncer de próstata en el Instituto de Oncología Maranhense Aldenora Bello. Las variables analizadas fueron: grupo etario, origen, ocupación, antígeno prostático específico inicial, escala de Gleason en la biopsia, escala de Gleason en la pieza quirúrgica (en pacientes cuyo tratamiento se incluyó la prostatectomía radical o resección transuretral de la próstata); y tratamiento establecido. Resultados: La franja etaria más afectada fue la de 71 a 80 años (39,7\%). La mayoría de los pacientes eran provenientes de São Luís - Maranhão (64,1\%) y sin ocupación (55,2\%). La mayor parte de los pacientes $(32,8 \%)$ tenía antígeno prostático específico inicial entre 4,1 y $10 \mathrm{ng} / \mathrm{ml}$. La escala de Gleason en la biopsia mostró que el $48,5 \%$ de los casos había desde tumores moderados hasta mal diferenciados. La terapia predominante consistió en tratamiento clínico y / o la orquidectomía subcapsular bilateral. Conclusión: El presente estudio demostró una asociación significativa del antígeno prostático específico con el grupo etario y con el Gleason, lo cual reveló una mayor tendencia de aumento de sus niveles cuando más avanzada fuera la edad y mayor fuera el grado histológico de los tumores. Del total de pacientes, 36,5\% obtuvieron escala de Gleason en la biopsia y en la pieza quirúrgica equivalentes. Palabras clave: Humanos; Masculino; Neoplasias de la Próstata; Adenocarcinoma; Factores Epidemiológicos; Biópsia 